\title{
Neues Weiterbildungsprogramm in Pharmazeutischer Medizin
}

\author{
Gabriel Schnetzler \\ Dr. med., FA Pharmazeutische Medizin
}

Im Rahmen der Akkreditierung der Medizinalberufe 2018 wurde eine Revision des Weiterbildungsprogramms in Pharmazeutischer Medizin eingeleitet, welches das SIWF am 12. März 2020 genehmigt und per 1. Juli 2020 in Kraft gesetzt hat [1]. Die Übergangsfrist ist gültig bis 30. Juni 2025 für Anwärterinnen und Anwärter, die sämtliche Bedingungen (exkl. Facharztprüfung) gemäss altem Programm (letzte Revision 14. April 2016) abgeschlossen haben.

\section{Zum Fachgebiet Pharmazeutische Medizin}

Die Pharmazeutische Medizin ist eine medizinischwissenschaftliche Disziplin, mit dem Ziel einer bestmöglichen therapeutischen Versorgung der Bevölkerung durch eine bedarfsgerechte Entwicklung von Heilmitteln (Arzneimittel und Medizinprodukte) [2] und deren optimalen Einsatz in der klinischen Praxis. Die Schweizerische Gesellschaft für Pharmazeutische Medizin wurde 1997 gegründet, und ein Weiterbildungsprogramm zum Erlangen des Facharzttitels ist seit dem 1. Januar 1999 akkreditiert. Mittlerweile haben über 120 Ärztinnen und Ärzte diesen erlangt [3]. Die fachärztliche Tätigkeit liegt in der Zusammenarbeit mit verschiedenen Disziplinen und Akteuren des

\section{Das Wichtigste in Kürze}

- Die Revision des Weiterbildungsprogramms in Pharmazeutischer Medizin tritt per 1. Juli 2020 in Kraft.

- Die Ausbildungsdauer bleibt unverändert fünf Jahre und unterteilt sich weiterhin in einen nichtfachspezifischen, patientenbezogenen klinischen Teil (mind. 2 Jahre) und einen fachspezifischen Teil an anerkannten Weiterbildungsstätten in Pharmazeutischer Medizin (2-3 Jahre).

- Neu wird für den Nachweis der fachspezifischen theoretischen Weiterbildung das Basisdiplom in Pharmazeutischer Medizin gemäss IFAPP/Pharmatrain Syllabus verlangt.

- Die wissensbasierten Lernziele wurden in definierte fachspezifische Kompetenzen überführt; die Kernpunktgebiete sind neu in sieben verschiedene Domänen aufgeteilt und widerspiegeln den Prozess der Heilmittelentwicklung und der therapeutischen Versorgung der Bevölkerung.

- Neu wird auch eine Publikation oder das Verfassen einer wissenschaftlichen Arbeit verlangt.
Gesundheitswesens im Rahmen der Entdeckung, Erforschung und Entwicklung von Heilmitteln, deren regulatorischer Zulassung und Erstattungsbeurteilung sowie deren praktischer und sicherer Anwendung im klinischen Alltag. Die patientenbezogene, evidenzbasierte Entscheidungsfindung hat einen direkten Einfluss auf die medizinische Versorgung zum Wohl und Nutzen der Patienten sowie der Gesellschaft, möglicherweise über die Landesgrenzen hinaus.

Eine bedarfsgerechte Heilmittelentwicklung unterliegt gesetzlichen, ethischen und qualitativen Anforderungen an die Planung und Durchführung von klinischen Studien und setzt eine korrekte Interpretation präklinischer, toxikologischer, pharmakologischer und klinischer Resultate unter Berücksichtigung des Nutzen-

\section{L'essentiel en bref}

- Le programme de formation postgraduée en médecine pharmaceutique entre en vigueur le 1er juillet 2020.

- La durée de la formation reste de cinq ans, avec la même répartition: une formation clinique non spécifique, axée sur les patients (minimum 2 ans); une formation spécifique en médecine pharmaceutique, dans des établissements reconnus (2 à 3 ans).

- Désormais, pour prouver le suivi d'une formation postgraduée théorique spécifique, le diplôme de base en médecine pharmaceutique, conforme aux formations de I'IFAPP / de Pharmatrain Syllabus est exigé.

- Les objectifs pédagogiques basés sur les connaissances ont été transformés en compétences spécifiques précises; les domaines de spécialisation sont désormais au nombre de sept et reflètent le processus de développement des médicaments et les soins thérapeutiques apportés à la population.

- A partir de maintenant, une publication ou la rédaction d'un travail scientifique sont aussi requis. 
Facharztprüfung Pharmazeutische Medizin (s. Ziffer 4)

Entueckung
und frühe

Wisssensvertiefung und Kompetenztraining an Weiterbildungsstätten basierend auf Swiss Catalogue of Core Competencies in Pharmaceutical Medicine [SC $\left.{ }^{3}-\mathrm{PM}\right]$ (s. Ziffer 3)

II I! I! II

Fachspezifische theoretische Weiterbildung (s. Ziffer 2.2.2):

Postgraduate Diploma in Pharmaceutical Medicine (30ECTS)

Nicht-fachspezifische, patientenbezogene klinische Weiterbildung (s. Ziffer 2.1.2)

* Mind. 1 Jahr Kat A/B; max. 1 Jahr Optionen (s. Ziffer 2.1.3)

Abbildung 1: Schematische Darstellung der Weiterbildung.

Risiko-Verhältnisses und sozioökonomischer Aspekte voraus. Zudem sind ein komplexes Wissen und praktische Erfahrung für den Aufbau und die Einreichung eines Zulassungsdossiers, eines Gesuchs um Aufnahme in die Vergütungslisten nach Kriterien der Wirksamkeit/Zweckmässigkeit/Wirtschaftlichkeit (WZW), für die kontinuierliche Weiterentwicklung von Heilmitteln auch nach der Registrierung, für die medizinische Beurteilung und Gewährleistung der Arzneimittelsicherheit durch adäquates Risikomanagement und Versorgungssicherheit sowie für die medizinisch-wissenschaftlichen Informationen zu Heilmitteln in der praktischen Anwendung erforderlich. All dies bedarf einer kompetenten Mitarbeit und Expertise von entsprechend qualifizierten Ärzten, weshalb Fachärzte für Pharmazeutische Medizin in verschiedenen Gebieten tätig sein können:

- in der translationalen Medizin und klinischen Forschung an Spitälern und akademischen Zentren (inkl. Clinical Trial Units),

- bei Herstellern und Vertreibern von pharmazeutischen, biomedizinischen Arzneimitteln und Heilvorrichtungen (inkl. Diagnostika) und deren Serviceanbietern,

- in unabhängigen Vertragsforschungsorganisationen (CRO),

- bei Zulassungs- und Überwachungsorganen (z.B. Swissmedic, BAG, Ethikkommissionen).

\section{Ziele der Weiterbildung}

Die Ausbildungsdauer bleibt unverändert fünf Jahre und unterteilt sich weiterhin in einen nichtfachspezi- fischen, patientenbezogenen klinischen Teil (mind. 2 Jahre) und einen fachspezifischen Teil an anerkannten Weiterbildungsstätten in Pharmazeutischer Medizin (2-3 Jahre, je nach Optionen, s. Abb. 1). Neu wird für den Nachweis der fachspezifischen theoretischen Weiterbildung das Basisdiplom in Pharmazeutischer Medizin gemäss IFAPP/Pharmatrain Syllabus [4] verlangt. Dabei handelt es sich um eine universitäre Weiterbildung im Umfang von mind. 30 ECTS, welche zum Beispiel am European Center for Pharmaceutical Medicine der Universität Basel als zweijähriger PostgraduateKurs angeboten wird.

Im Zuge der Revision wurden die wissensbasierten Lernziele in definierte fachspezifische Kompetenzen überführt [3]. Die Kernpunktgebiete sind neu in sieben verschiedene Domänen aufgeteilt und widerspiegeln den Prozess der Heilmittelentwicklung und der therapeutischen Versorgung der Bevölkerung. Der Swiss Catalogue of Core Competencies in Pharmaceutical Medicine ( $\left.\mathrm{SC}^{3}-\mathrm{PM}\right)$ beschreibt das angewandte Wissen, die Fähigkeiten und das erwartete Verhalten zum Ende der Facharztausbildung und ist mit internationalen Standards [5, 6] abgeglichen. Der Katalog ist auf der Website der SGPM (www.sgpm.ch) abrufbar.

\section{Neue Bedingung einer Publikation oder wissenschaftlichen Arbeit}

Mit der Revision 2020 wird neu auch eine Publikation oder das Verfassen einer wissenschaftlichen Arbeit verlangt. Dies erfolgt in Anlehnung an die im $\mathrm{SC}^{3}$-PM beschriebenen Kompetenzen. Dabei gelten die Vorgaben des SIWF bezüglich Umfang, Format, Autorenschaft und 
Tabelle 1: Kernkompetenzen des Facharztes für Pharmazeutische Medizin.

\begin{tabular}{|c|c|c|}
\hline Domäne & Kompetenzen & $\mathrm{SC}^{3}-\mathrm{PM}$ \\
\hline $\begin{array}{l}\text { Entdeckung und } \\
\text { frühe Entwicklung }\end{array}$ & $\begin{array}{l}\text { Kann ungedeckten therapeutischen Bedarf («Unmet Medical Need») identifizieren, kann } \\
\text { die Evidenz neuer Heilmittelmöglichkeiten für die klinische Entwicklung evaluieren und } \\
\text { kann ein klinisches Entwicklungsprogramm mit definiertem Produktprofil («Target Pro- } \\
\text { duct Profile») erstellen. }\end{array}$ & C1-C9 \\
\hline $\begin{array}{l}\text { Klinische Entwicklung } \\
\text { und klinische Studien }\end{array}$ & $\begin{array}{l}\text { Kann explorative und bestätigende klinische Studien erstellen, als Sponsor/-Prüfarzt } \\
\text { durchführen und auswerten sowie die damit verbundenen Studienberichte und Manu- } \\
\text { skripte zur Veröffentlichung und Einreichung bei Behörden verfassen. }\end{array}$ & $\mathrm{C} 10-\mathrm{C} 17$ \\
\hline $\begin{array}{l}\text { Pharmakovigilanz und } \\
\text { Risiko-Management }\end{array}$ & $\begin{array}{l}\text { Kann die Anwendung und Auswertung von verschiedenen Methoden zur Arzneimittel- } \\
\text { überwachung nach der Zulassung («post-authorization surveillance») evaluieren, um die } \\
\text { Anforderungen von nationalen/internationalen Behörden für angemessene Information } \\
\text { und Risikominimierung für Patienten und Studienversuchspersonen zu erreichen. }\end{array}$ & $\mathrm{C} 28-\mathrm{C} 35$ \\
\hline $\begin{array}{l}\text { Sozioökonomie des } \\
\text { Gesundheitswesens }\end{array}$ & $\begin{array}{l}\text { Kann den sinnvollen Einsatz von diagnostischen, prophylaktischen und therapeutischen } \\
\text { Mitteln bei der Betreuung von Gesunden und Kranken bewerten und unterstützt dabei } \\
\text { den optimalen Einsatz der zur Verfügung stehenden Mittel unter Berücksichtigung der } \\
\text { gesetzlichen Grundlagen. }\end{array}$ & $\mathrm{C} 43-\mathrm{C} 45$ \\
\hline $\begin{array}{l}\text { Kommunikation und } \\
\text { Management }\end{array}$ & $\begin{array}{l}\text { Kann effektive Kommunikationsfähigkeiten und Managementtechniken für die Zusam- } \\
\text { menarbeit zwischen den verschiedenen Akteuren im Gesundheitssystem einschliesslich } \\
\text { Klinik, Behörden und Institutionen einsetzen. }\end{array}$ & C46-C54 \\
\hline
\end{tabular}

Peer-Review-Prozess. Das Thema der Publikation wie auch einer Dissertation muss nicht im Fachgebiet des angestrebten Titels liegen. Zusätzlich wird auch ein medizinisch-wissenschaftliches, regulatorisch relevantes Dokument zuhanden einer kompetenten Behörde akzeptiert, sofern es denselben Umfang hat. Hierzu können Studienprotokolle oder -berichte, Safety Reports oder Teile einer Zulassungseinreichung gezählt werden.

\section{Engere Zusammenarbeit zwischen Fachge- sellschaft und den Weiterbildungsstätten}

Im Zuge des Akkreditierungsprozesses wurde von der SGPM das Weiterbildungsstättenleiter-Kränzli ins Leben gerufen. Dieses ermöglicht die engere Zusammenarbeit zwischen der Fachgesesellschaft sowie unter den einzelnen Weiterbildungsstätten. So können spezifische Expertisen der verschiedenen Stätten (z.B. Akademie, Industrie, Behörden) durch gemeinsame Trainings genutzt und die Qualität der Weiterbildung durch Erfahrungsaustausch verbessert werden.

Die SGPM beteiligt sich zudem an der Organisation von zwei jährlichen Fachveranstaltungen für die Weiterund Fortbildung: dem Spring Meeting der Schweizerischen Gesellschaft für Pharmakologie und Toxikologie sowie dem Herbstsymposium zusammen mit der Swiss Association of Pharmaceutical Professionals (www.SwAPP.ch). Die wissenschaftliche Tätigkeit von
Weiterzubildenden soll durch Posterpreise der SGPM zusätzlich gefördert werden.

Die Weiterbildung zum Facharzttitel Pharmazeutische Medizin bietet sich auch für die Zukunft als eine attraktive Option für Kandidatinnen und Kandiaten an, die eine berufliche Laufbahn in der klinischen Forschung, Heilmittelindustrie oder bei Gesundheitsbehörden anvisieren.

Literatur

1 https://www.siwf.ch/weiterbildung/facharzttitel-und-schwerpunkte/pharmazeutische-medizin.cfm

2 Heilmittel werden bei der Erkennung, Verhütung und Behandlung von Krankheiten, Verletzungen und Behinderung eingesetzt. Der Begriff Heilmittel beinhaltet Arzneimittel und Medizinprodukte (https://www.admin.ch/opc/de/classifiedcompilation/20002716/index.html)

3 Schnetzler G, Bremgartner MF, Grossmann Straessle R, Spirk D, Tay F, Troxler Saxer R, Traber M. Evolution to a CompetencyBased Training Curriculum for Pharmaceutical Medicine Physicians in Switzerland. Front Pharmacol. 2019 Feb 27;10:164. doi: 10.3389/fphar.2019.00164

4 https://www.pharmatrain.eu/guidelines.php $\rightarrow$ Syllabus \& Outcomes

5 Kerpel-Fronius S, Becker S, Barrett J, Brun J, Carlesi R, Chan A, Collia LF, Dubois DJ, Kleist P, Koski G, Kurihara C, Laranjeira LF, Schenk J, Silva H. The Shared Ethical Responsibility of Medically and Non-medically Qualified Experts in Human Drug Development Teams. Front Pharmacol. 2018 Sep 3;9:843. doi: 10.3389/fphar.2018.00843

6 Stonier PD, Silva H, Boyd A, Criscuolo D, Gabbay FJ, Imamura K, Kesselring G, Kerpel-Fronius S, Klech H, Klingmann I. Evolution of the Development of Core Competencies in Pharmaceutical Medicine and Their Potential Use in Education and Training. Front Pharmacol. 2020 Mar 19;11:282. doi: 10.3389/fphar.2020.00282 